\title{
INFLUÊNCIA DO MÉTODO DE PREPARAÇÃo DE ELETROCATALISADORES PtRu/C SOBRE A ATIVIDADE CATALÍTICA FRENTE À REAÇÃO DE OXIDAÇÃO DE ETANOL EM MEIO ÁCIDO
}

\author{
Walber dos Santos Gomes, Uriel Lean Valente Silva e José Pio Iúdice de Souza* \\ Faculdade de Química, Instituto de Ciências Exatas e Naturais, Universidade Federal do Pará, Rua Augusto Corrêa, 1, 66075-110 \\ Belém - PA, Brasil
}

Recebido em 29/8/12; aceito em 25/10/12; publicado na web em 18/2/13

\begin{abstract}
INFLUENCE OF METHOD OF PREPARATION OF PtRu/C ELECTROCATALYSTS ON THE CATALYTIC ACTIVITY FOR THE ETHANOL OXIDATION REACTION IN ACIDIC MEDIUM. In this work the influence of variations in the borohydrate reduction method on the properties of PtRu/C electrocatalysts was investigated. The electrocatalysts were prepared using $1: 1 ; 2: 1 ; 5: 1 ; 50: 1$ and 250:1 molar ratios of $\mathrm{NaBH}_{4}$ to metals. The reduction was also performed by dripping or by fast addition of the solution. The results showed that PtRu nanoparticles obtained by fast addition had the smallest crystallite sizes. It was also noted that the catalytic activity increased as the borohydrate:metal molar ratio increased. The PtRu/C electrocatalyst (50:1) obtained by fast addition presented the best catalytic activity for ethanol electro-oxidation.
\end{abstract}

Keywords: $\mathrm{NaBH}_{4}$; PtRu; ethanol oxidation.

\section{INTRODUÇÃO}

Durante os últimos anos tem havido um ressurgimento do interesse no desenvolvimento das tecnologias das células a combustível. ${ }^{1,2}$ Entre as diferentes opções, as células a combustível de membrana trocadora de prótons (PEMFC) têm adquirido um status privilegiado devido a sua versatilidade para aplicação em um grande número de diversos aparelhos portáteis e na indústria automotiva. ${ }^{3,4}$ Neste tipo de célula a combustível, inicialmente, a maioria das pesquisas sempre esteve direcionada mais para aplicação do hidrogênio como combustível. Entretanto, algumas questões relativas ao armazenamento e transporte seguros do hidrogênio ainda não foram satisfatoriamente resolvidas e continuam sendo intensamente pesquisadas., 5,6

Nesse sentido, as células que utilizam álcool diretamente como combustível (DAFC- Direct Alcohol Fuel Cell) vêm ganhando notoriedade e despertando interesse, pois além de não haver necessidade de produção e armazenamento de hidrogênio, já existe uma infraestrutura para distribuição destes combustíveis. ${ }^{7,8}$ As DAFCs são bastante promissoras para possíveis aplicações móveis (carros, bicicletas, etc.), portáteis (notebooks, tablets, telefones celulares, etc.) e estacionárias. ${ }^{9}$

Dentre os combustíveis alternativos, o etanol é mais seguro e tem maior densidade de energia que o metanol $\left(8,01 \mathrm{kWh} \mathrm{kg}^{-1}\right.$ contra $\left.6,09 \mathrm{kWh} \mathrm{kg}^{-1}\right)$. Além disso, o etanol é facilmente produzido pela fermentação do açúcar contido em materiais brutos. ${ }^{8}$ Quando o etanol se oxida completamente os produtos finais são $\mathrm{CO}_{2}$ e água. ${ }^{10}$

As células a combustível que operam em temperaturas baixas (menor que $100{ }^{\circ} \mathrm{C}$ ) precisam de catalisadores eficientes para promover as reações eletródicas. Nestes tipos de células, tanto para as reações anódicas, quanto para as catódicas, a platina tem-se mostrado um elemento essencial para obtenção de catalisadores com desempenho aceitável. ${ }^{11,12}$

\section{Catalisadores binários $\mathrm{PtRu} / \mathrm{C}$ e redução via boro-hidreto}

Para um determinado eletrodo sólido o aumento da sua atividade catalítica está diretamente relacionado à diminuição do sobrepotencial

*e-mail: jpio@ufpa.br da reação de oxidação de alcoóis. ${ }^{13,14}$ Também, o aumento da eficiência faradáica está relacionado à habilidade dos catalisadores em oxidar completamente o combustível a $\mathrm{CO}_{2}$, ou seja, está relacionado à seletividade do catalisador. No caso do etanol, o ácido acético e o acetaldeído são obtidos como produtos da oxidação parcial, os quais correspondem a um número de 4 e 2 elétrons por molécula, respectivamente, sendo que a oxidação completa do etanol envolve 12 elétrons.

Até agora, o uso de platina é inevitável para a adsorção e oxidação de alcoóis em meio ácido, porém, lamentavelmente, a oxidação de alcoóis leva à formação de espécies CO adsorvido, a qual envenena a superfície catalítica. Para contornar esse problema, duas estratégias foram desenvolvidas em paralelo: a dispersão da platina a nível nanoescalar e a modificação da platina pela adição de um elemento metálico diferente. ${ }^{12}$

Um exemplo da segunda estratégia é a adição de rutênio à platina (cocatalisador), o que permite a formação de espécies oxigenadas em potenciais inferiores a $0,25 \mathrm{~V}$, facilitando a oxidação de $\mathrm{CO}$ a $\mathrm{CO}_{2}$. Quando se utiliza somente platina, as espécies oxigenadas são formadas em potenciais acima de $0,8 \mathrm{~V}$, diminuindo a eficiência do processo. ${ }^{15} \mathrm{~A}$ forte adsorção de $\mathrm{CO}$ nos sítios de $\mathrm{Pt}$ faz com que a corrente elétrica no anodo seja originada da oxidação do combustível, que ocorre apenas nas poucas regiões livres de CO. Estas áreas se formam ao redor dos átomos do outro metal, sendo que o restante de área de Pt permanece coberto por uma monocamada compacta de $\mathrm{CO}$ adsorvido. ${ }^{16,17}$

A liga PtRu é considerada o catalisador com mais alta tolerância ao CO. O aumento na atividade catalítica é atribuído ao "mecanismo bifuncional" e ao efeito eletrônico. ${ }^{18}$

No mecanismo bifuncional, propõe-se que a espécie reagente $\left(\mathrm{H}_{2}\right.$, $\mathrm{CH}_{3} \mathrm{OH}, \mathrm{CH}_{3} \mathrm{CH}_{2} \mathrm{OH}$ ) e o contaminante se adsorvem preferencialmente nos átomos de Pt, enquanto que o outro metal, menos nobre e mais oxidável, produz espécies oxigenadas ou óxidos hidratados que atuam diretamente na oxidação do contaminante através de reação tipo Langmuir-Hinshelwood. ${ }^{19,20}$ A vantagem é que a nucleação de espécies oxigenadas no segundo metal ocorre em potenciais eletródicos menos positivos em relação à platina pura, ou seja, o papel do segundo metal baseia-se na relativa facilidade que apresenta de formar óxidos hidratados em sobrepotenciais mais baixos que a platina. Desta forma, essas espécies oxigenadas desempenham o papel de agentes 
oxidantes, convertendo o $\mathrm{CO}$ quimissorvido na platina a dióxido de carbono, que rapidamente se dessorve da superfície do catalisador. ${ }^{21}$

Alguns autores sugerem que a formação de óxidos, como o $\mathrm{RuO}_{2} \mathrm{e}$ o $\mathrm{RuO}_{3}$, por oxidação da superfície do $\mathrm{Ru}$, causaria perda da atividade eletrocatalítica do catalisador PtRu, pois essas espécies não são ativas como doadores de oxigênio para a oxidação de $\mathrm{CO} .{ }^{22}$

Por outro lado, estudos realizados por Rolison e colaboradores ${ }^{23}$ através de análises termogravimétricas e espectroscopia fotoeletrônica de raio-x (XPS) em catalisadores PtRu comerciais revelaram a presença de quantidades substanciais de óxido de rutênio hidratado $\left(\mathrm{RuOH} \cdot \mathrm{xH}_{2} \mathrm{O}\right)$. Segundo esses autores, essa mistura de fases $(\mathrm{RuOH}$. $\mathrm{xH}_{2} \mathrm{O}$, Pt metálica, óxido de Pt e Ru metálico) levaria a um aumento na atividade eletrocatalítica em relação às ligas de PtRu. Essa proposta se baseia no fato de que o $\mathrm{RuO}_{2}$ se protonaria eletroquimicamente através de uma reação rápida e reversível.

$\mathrm{O}$ efeito eletrônico, ou mecanismo intrínseco, postula que a presença do segundo metal causa uma mudança do centro de banda $d$ da platina, sendo que a posição do centro de banda $d$ é uma medida da energia de adsorção de um adsorbato (por exemplo, CO). Quanto maior a energia do centro da banda $d$, maior será a interação $\mathrm{Pt}-\mathrm{CO}$. O aumento da tolerância ao $\mathrm{CO}$ para $\mathrm{PtRu}$ em relação à $\mathrm{Pt}$ pura pode ser atribuído a uma menor energia de adsorção de CO sobre $\mathrm{Pt}$, em razão desta mudança do centro de banda $d$ da platina. $\mathrm{O}$ efeito da adição de um segundo elemento (metal) à platina tem sido analisado por espectroscopia de absorção de raio-x (XAS) in situ, sendo observado que os átomos de platina na liga apresentam alterações tanto no ordenamento atômico (distância da ligação Pt-Pt, número de coordenação, etc.) como nos níveis eletrônicos (ocupação da banda 5-d) quando comparado ao sistema que utiliza Pt pura nas mesmas condições. ${ }^{24,25}$ Estes fatores podem influenciar as energias de quimissorção de $\mathrm{H}_{2}$ e de $\mathrm{CO}$, contribuindo para menor ação do veneno catalítico sobre a cinética da reação eletródica de interesse. ${ }^{26}$

A estrutura cristalina da platina é cúbica de face centrada (CFC) enquanto a do rutênio é hexagonal de empacotamento compacto. Para frações atômicas até $0,7(\mathrm{Ru} / \mathrm{Pt})$, platina e rutênio formam solução sólida com átomos de rutênio substituindo átomos de platina em pontos da estrutura cúbica de face centrada. $\mathrm{O}$ parâmetro de rede diminui de 3,923 ̊ (Pt pura) para 3,83 A, até uma composição com $70 \%$ de $\mathrm{Ru}$. Acima desta proporção de Ru, outras soluções sólidas são formadas, com átomos de Pt substituindo átomos de Ru em uma estrutura hexagonal de empacotamento compacto (hcp). ${ }^{27}$

De acordo com Zhou e colaboradores, ${ }^{7}$ a formação de liga de platina com um segundo ou um terceiro metal, tais como, Pt-Ru, Pt-Sn, Pt-Ni, Pt-Ru-W, Pt-Ru-Rh, etc, é fundamental para a modificação de suas propriedades catalíticas e consequente superação do envenenamento por intermediários. Em eletrodos para células a combustível $(\mathrm{CaC})$, estes catalisadores são suportados em carbono na tentativa de aumentar a área e diminuir a quantidade de metal nobre usado na sua confecção. ${ }^{27}$

Estudos recentes da oxidação eletroquímica do etanol estão centrados no desenvolvimento de catalisadores apropriados, entre os citados anteriormente, as ligas de PtRu, PtSn e PtRuSn são recomendadas como as mais adequadas. ${ }^{27}$ Usando-se DEMS tem sido demonstrado que catalisadores $\mathrm{PtRu}$ de diferentes composições reduzem o inicio de oxidação do etanol a $\mathrm{CO}_{2}$ e acetaldeído. ${ }^{28}$

A preparação de novos catalisadores dispersos em materiais condutores com alta área superficial, como os diversos materiais de carbono, propicia um aumento na interface catalisador - eletrólito, o que possibilita a diminuição da carga de platina nas células a combustivel. Um bom suporte deve fornecer uma estrutura que permita a condução eletrônica e aumente a dispersão da fase ativa. Tanto a natureza química dos componentes como o método de preparo do catalisador são fatores indispensáveis no desenvolvimento de catalisadores mais ativos. ${ }^{21}$

Os catalisadores $\mathrm{Pt} / \mathrm{C}$ e $\mathrm{PtRu} / \mathrm{C}$ são preparados por diversos métodos; técnicas utilizando coloides, organometálicos ou complexos de coordenação, redução química de sais precursores e deposição metálica por técnicas eletroquímicas resumem as rotas mais relevantes de preparação que vêm sendo publicadas. ${ }^{29}$

No método da impregnação são geralmente utilizados como redutores o etilenoglicol, ácido fórmico e $\mathrm{NaBH}_{4} \cdot{ }^{30}$ Muitos estudos têm mostrado que a atividade dos eletrocatalisadores PtRu é bastante influenciada por diversos fatores relacionados ao método de preparação. A variação da proporção molar entre o agente redutor boro-hidreto de sódio e os metais é um destes fatores que foi estudado no método de impregnação. ${ }^{31}$

O método do boro-hidreto de sódio é bastante estudado e utilizado na síntese de eletrocatalisadores para aplicação em células a combustível. O boro-hidreto de sódio $\left(\mathrm{NaBH}_{4}\right)$ é encontrado, geralmente, na forma de um sólido branco (em pó ou pelotas), inodoro, com boa solubilidade em metanol (13 g/100 mL), etanol $(3,16 \mathrm{~g} / 100 \mathrm{~mL})$ e água $(54 \mathrm{~g} / 100 \mathrm{~mL})$. Em metanol e etanol, apesar de solúvel, decompõe-se em boratos. Em água é estável em pH 14, decompondo-se rapidamente em soluções neutras e ácidas. Trata-se de um agente redutor relativamente forte, bastante utilizado na produção de compostos orgânicos e inorgânicos. O método de redução via boro-hidreto é de simples execução e, relativamente, reprodutível, além da sua eficácia comprovada na produção de nanopartículas. ${ }^{11} \mathrm{As}$ reações envolvidas na síntese de um eletrocatalisador $\mathrm{PtRu} / \mathrm{C}$ pelo método do boro-hidreto, utilizando $\mathrm{RuCl}_{3} \mathrm{e}_{2} \mathrm{PtCl}_{6}$ como precursores metálicos, podem ser resumidas pelas equações:

$8 \mathrm{Ru}^{3+}+3 \mathrm{BH}_{4}^{-}+12 \mathrm{H}_{2} \mathrm{O} \rightarrow 8 \mathrm{Ru}+3 \mathrm{~B}(\mathrm{OH})_{4}{ }^{-}+24 \mathrm{H}^{+}$

$2 \mathrm{PtCl}_{6}{ }^{2-}+\mathrm{BH}_{4}^{-}+4 \mathrm{H}_{2} \mathrm{O} \rightarrow 2 \mathrm{Pt}+\mathrm{B}(\mathrm{OH})_{4}^{-}+8 \mathrm{H}^{+}+12 \mathrm{Cl}^{-}$

Considerando o exposto acima, este trabalho visou verificar a influência da razão molar entre o redutor $\mathrm{NaBH}_{4}$ e os sais precursores de $\mathrm{Pt}$ e Ru, bem como do método de adição do redutor, por gotejamento ou adição rápida, sobre as propriedades dos eletrocatalisadores $\mathrm{PtRu}$, suportados em carbono Vulcan XC-72R, e testar suas atividades catalíticas frente à reação de oxidação de etanol em meio ácido.

\section{PARTE EXPERIMENTAL}

\section{Preparação dos eletrocatalisadores PtRu/C}

Foi utilizado como suporte o carbono black Vulcan XC-72R (de área superficial em torno de $240 \mathrm{~m}^{2} \mathrm{~g}^{-1}$ determinada por BET). O pó de carbono foi suspenso em água ultrapura e sonicado. Os sais precursores, $\mathrm{H}_{2} \mathrm{PtCl}_{6} \cdot 6 \mathrm{H}_{2} \mathrm{O}$ (Acros) e $\mathrm{RuCl}_{3} \cdot 3 \mathrm{H}_{2} \mathrm{O}$ (Acros), foram dissolvidos em água ultrapura e levados ao banho ultrassom. Estas duas soluções foram misturadas e agitadas suficientemente. A quantidade de metal precursor foi ajustada para uma razão molar 1:1 e o total de metal no catalisador foi de $40 \%(\mathrm{~m} / \mathrm{m})$. Soluções de diferentes concentrações de boro-hidreto de sódio $\left(\mathrm{NaBH}_{4}\right)$ (Sigma-Aldrich) foram preparadas, nas quais as razões molares entre o $\mathrm{NaBH}_{4}$ e os metais $\mathrm{Pt}$ e $\mathrm{Ru}$ foram de $1,2,5,50$ e 250 . As soluções de $\mathrm{NaBH}_{4}$ foram primeiramente gotejadas nas misturas contendo carbono Vulcan e os íons metálicos a $80^{\circ} \mathrm{C}$ (obtiveram-se os catalisadores $\mathrm{PtRu} / \mathrm{C}-\mathrm{X}-(\mathrm{GG}), \mathrm{X}$ é a razão molar). Posteriormente, o mesmo procedimento foi utilizado para outro conjunto de catalisadores, porém adicionando-se rapidamente as soluções de $\mathrm{NaBH}_{4}$ (obtiveram-se os catalisadores PtRu/C-X-(AR), $\mathrm{X}$ é a razão molar). Após $30 \mathrm{~min}$ os catalisadores preparados foram filtrados e lavados. Os catalisadores foram então secados e mantidos em estufa a $80^{\circ} \mathrm{C}$ por $12 \mathrm{~h}$. 


\section{Caracterização física dos eletrocatalisadores}

As análises de energia dispersiva de raios-X (EDX) foram realizadas em um equipamento de fluorescência da Shimadzu, modelo EDX-700, série Rayny, equipado com uma fonte de ródio.

As medidas de difração de raios-X (DRX) foram realizadas num difratômetro da PANalytical, modelo X'PERT PRO MPD (PW3040/60), com goniômetro PW3050/60 ( $\theta / \theta)$ e tubo de raios-X cerâmico de ânodo de $\mathrm{Cu}(\mathrm{K} \alpha 1$ 1,540598 Å), modelo PW3373/00, foco fino longo, $2200 \mathrm{~W}, 60 \mathrm{kv}$.

\section{Medidas eletroquímicas}

Os catalisadores foram testados em eletrodos de camada ultrafina através das técnicas de voltametria cíclica e cronoamperometria. Foram utilizados um eletrodo reversível de hidrogênio (ERH) e uma placa de Pt, como eletrodos de referência e auxiliar, respectivamente. Foi utilizado o potenciostato PGSTAT320N Autolab acoplado a um microcomputador.

Os voltamogramas cíclicos foram obtidos, primeiramente, em soluções de $0,5 \mathrm{~mol} \mathrm{~L}^{-1}$ de $\mathrm{H}_{2} \mathrm{SO}_{4}$ e depois em soluções de $0,5 \mathrm{~mol} \mathrm{~L}^{-1}$ $\mathrm{H}_{2} \mathrm{SO}_{4}+0,5 \mathrm{~mol} \mathrm{~L}^{-1} \mathrm{CH}_{3} \mathrm{CH}_{2} \mathrm{OH}$. Durante o experimento foi mantido fluxo de $\mathrm{N}_{2(\mathrm{~g})}$. As curvas voltamétricas foram obtidas na velocidade de varredura de potencial de $20 \mathrm{mV} \mathrm{s}^{-1}$. A faixa de potencial para o estudo foi fixada entre 0,1 e $1,0 \mathrm{~V}$, com o objetivo de evitar alterações na composição superficial dos eletrocatalisadores.

Os experimentos cronoamperométricos foram realizados à temperatura ambiente nas mesmas soluções em que foram realizadas as voltametrias cíclicas. Foi aplicado o potencial de $0,5 \mathrm{~V}$ por $20 \mathrm{~min}$.

Em ambas as técnicas os valores de corrente foram normalizados pela carga de oxidação de uma monocamada de CO adsorvido, conforme descrito em Souza et al. ${ }^{32}$

\section{RESSULTADOS E DISCUSSÃO}

\section{EDX e DRX dos eletrocatalisadores}

Os difratogramas de raio-x mostram (Figuras 1 e 2) que os picos de difração dos catalisadores, obtidos por ambos os métodos, foram ligeiramente deslocados para ângulos maiores que o da platina, mostrando que a platina formou uma solução sólida com átomos Ru substituindo átomos de Pt na estrutura cúbica de face centrada do cristal de platina. ${ }^{7}$

Observa-se nos difratogramas dos eletrocatalisadores de $\mathrm{PtRu} / \mathrm{C}$ que existe uma redução da intensidade do pico relativo à face (200), o que indica um maior grau de ligamento entre a platina e o rutênio, conforme observado por Hyun e colaboradores, ${ }^{31}$ que sintetizaram eletrocatalisadores $\mathrm{Pt}_{1} \mathrm{Ru}_{1} / \mathrm{C}$ utilizando concentrações de $\mathrm{NaBH}_{4}$ seguindo a ordem de proporções molares entre o $\mathrm{NaBH}_{4}$ e os metais de $1,2,5,15,50$ e 250 .

Os picos de difração de PtRu/C-5(GG), PtRu/C-50(GG) e PtRu/C250(GG) nesta face são menos intensos ou mais largos do que em PtRu/C-1(GG) e PtRu/C-2(GG) (Figura 1). O mesmo se observa para os sistemas $\mathrm{PtRu} / \mathrm{C}$ obtidos por adição rápida, à exceção de PtRu/C-250(AR) (Figura 2).

Os tamanhos médios de cristalitos encontrados para os eletrocatalisadores PtRu/C-1(GG), PtRu/C-2(GG), PtRu/C-5(GG), PtRu/C-50(GG) e PtRu/C-250(GG) estão na faixa de 2,6 a 4,3 nm. Para os eletrocatalisadores PtRu/C-1(AR), PtRu/C-2(AR), PtRu/C-5(AR), PtRu/C-50(AR) e PtRu/C-250(AR) estão na faixa de 1,8 a $3,9 \mathrm{~nm}$.

De acordo com estudos anteriores, Hyun e colaboradores ${ }^{31}$ encontraram valores na ordem de 2,2 - 4,1nm para $\mathrm{PtRu} / \mathrm{C}$, porém, a metodologia utilizada foi o despejamento rápido da suspensão de

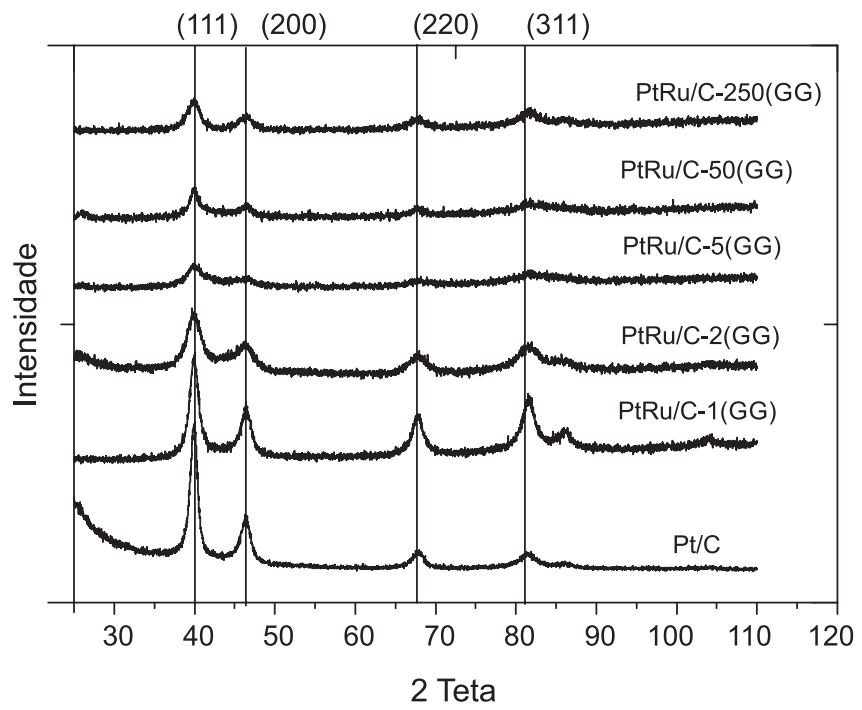

Figura 1. Difratogramas de raios $X$ dos eletrocatalisadores PtRu/C-1, PtRu/C-2, PtRu/C-5, PtRu/C-50 e PtRu/C-250 obtidos por gotejamento de solução de $\mathrm{NaBH}_{4}$

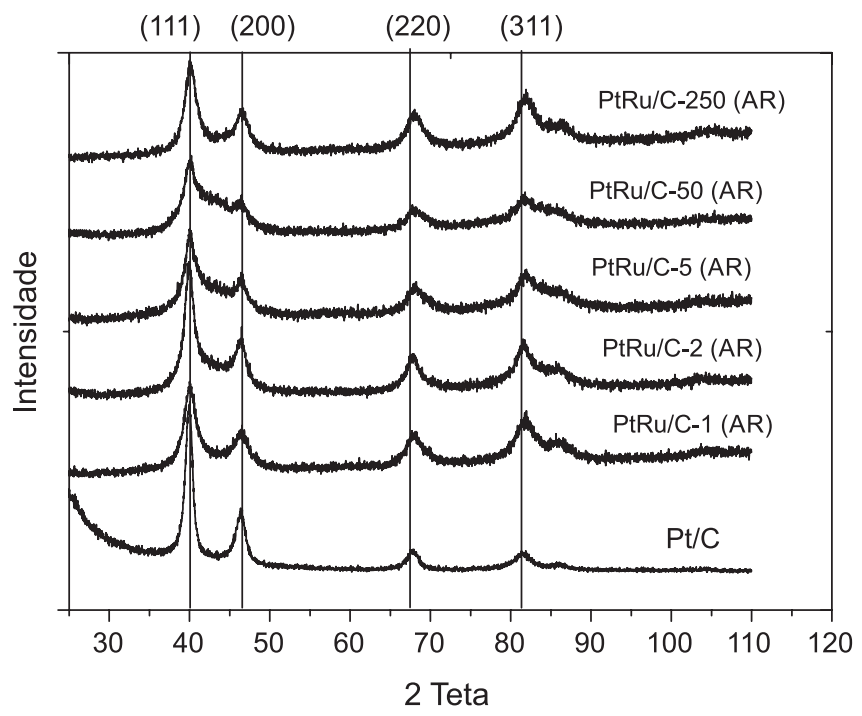

Figura 2. Difratogramas de raios $X$ dos eletrocatalisadores $P t R u / C-1$, PtRu/C-2, PtRu/C-5, PtRu/C-50 e PtRu/C-250 obtidos por adição rápida de solução de $\mathrm{NaBH}_{4}$

carbono, contendo os sais precursores, sobre as diferentes soluções de $\mathrm{NaBH}_{4}$, ou seja, procedimento inverso ao realizado neste trabalho.

Brandalise e colaboradores ${ }^{11}$ sintetizaram eletrocatalisadores PtRu na proporção nominal 50:50 por meio de boro-hidreto de sódio e encontraram tamanho médio das partículas de $3 \mathrm{~nm}$, sendo a composição obtida por EDX de PtRu 57:43. O valor obtido neste trabalho, para uma composição PtRu 56:44, foi de $3,8 \mathrm{~nm}$, porém, não há especificação da concentração de $\mathrm{NaBH}_{4}$.

Kashyout e colaboradores ${ }^{33}$ trabalharam com diferentes tempos de redução com etanol em refluxo para obtenção dos eletrocatalisadores PtRu/C1, PtRu/C2 e PtRu/C4, onde os números 1, 2 e 4 se referem ao tempo em horas de refluxo à temperatura de $\left(80 \pm 2{ }^{\circ} \mathrm{C}\right)$. $\mathrm{O}$ tamanho dos cristalitos obtidos foi $4,9 \mathrm{~nm}$ para $\mathrm{PtRu} / \mathrm{C} 1, \mathrm{PtRu} / \mathrm{C} 2 \mathrm{e}$ 4,7 nm para $\mathrm{PtRu} / \mathrm{C} 4$. A comparação da metodologia usada com a de redução via boro-hidreto, nos trabalhos já citados e os resultados ora apresentados, sugere que podem ser obtidos tamanhos de cristalitos menores via redução por boro-hidreto. 
As Tabelas 1 e 2 apresentam os valores para as razões atômicas nominais de partida, razões atômicas obtidas e tamanho médio dos cristalitos obtidos através de difratogramas de raio-x, utilizando a Equação de Debye-Sherrer, para os eletrocatalisadores obtidos por gotejamento e por adição rápida de soluções com diferentes concentrações de $\mathrm{NaBH}_{4}$.

Tabela 1. Razão atômica (análises de EDX ) e tamanho médio de cristalito (Equação de Scherrer) dos catalisadores preparados por gotejamento

\begin{tabular}{lcccc}
\hline Catalisadores & $\begin{array}{c}\text { Composição } \\
\text { nominal }\end{array}$ & $\begin{array}{c}\text { Razão } \\
\text { atômica }\end{array}$ & $\begin{array}{c}\text { Tamanho } \\
\text { médio de cris- } \\
\text { talito (nm) }\end{array}$ & $\begin{array}{c}\text { Parâmetro } \\
\text { de retículo } \\
(\AA)\end{array}$ \\
\hline Pt & - & - & 5,7 & 3,906 \\
PtRu/C-1 & $50: 50$ & $52: 48$ & 4,3 & 3,873 \\
PtRu/C- 1 & $50: 50$ & $56: 44$ & 3,8 & 3,876 \\
PtRu/C-5 & $50: 50$ & $53: 47$ & 2,8 & 3,879 \\
PtRu/C-50 & $50: 50$ & $43: 57$ & 2,7 & 3,889 \\
PtRu/C-250 & $50: 50$ & $45: 55$ & 2,6 & 3,891 \\
\hline
\end{tabular}

Tabela 2. Razão atômica (análises de EDX) e tamanho médio de cristalito (Equação de Scherrer) dos catalisadores preparados por adição rápida

\begin{tabular}{lcccc}
\hline Catalisadores & $\begin{array}{c}\text { Composição } \\
\text { nominal }\end{array}$ & $\begin{array}{c}\text { Razão } \\
\text { atômica }\end{array}$ & $\begin{array}{c}\text { Tamanho } \\
\text { médio de cris- } \\
\text { talito }(\mathrm{nm})\end{array}$ & $\begin{array}{c}\text { Parâmetro } \\
\text { de reticulo } \\
(\AA)\end{array}$ \\
\hline $\mathrm{Pt}$ & - & - & 5,7 & 3,906 \\
$\mathrm{PtRu} / \mathrm{C}-1$ & $50: 50$ & $48: 52$ & 3,9 & 3,883 \\
PtRu/C- 1 & $50: 50$ & $44: 56$ & 3,8 & 3,886 \\
PtRu/C-5 & $50: 50$ & $43: 57$ & 2,7 & 3,889 \\
PtRu/C-50 & $50: 50$ & $49: 51$ & 1,8 & 3,892 \\
PtRu/C-250 & $50: 50$ & $48: 52$ & 1,8 & 3,892 \\
\hline
\end{tabular}

Na Tabela 1 observa-se que para baixas concentrações de $\mathrm{NaBH}_{4}$ (proporções molares entre $\mathrm{NaBH}_{4}$ e metais Pt e Ru, 1, 2 e 5) a quantidade de platina no eletrocatalisador está acima dos valores nominais de partida. No entanto, a quantidade de platina não é mantida acima dos valores nominais com o aumento da concentração de $\mathrm{NaBH}_{4}$ (proporções molares entre $\mathrm{NaBH}_{4}$ e metais Pt e Ru, 50 e 250) como mostram as composições obtidas para os eletrocatalisadores PtRu/C50(GG) e PtRu/C-250(GG), em que a platina apresenta valores abaixo das composições nominais de partida.

A Tabela 2 mostra que para todas as concentrações de $\mathrm{NaBH}_{4}$, a proporção atômica de platina obtida por EDX, em todos os eletrocatalisadores $\mathrm{PtRu} / \mathrm{C}(\mathrm{AR})$, foi sempre muito próxima do valor nominal de partida, não havendo qualquer alteração significativa com o aumento da concentração de $\mathrm{NaBH}_{4}$

Os catalisadores obtidos por ambos os métodos mencionados neste trabalho mostram uma diminuição no tamanho médio de cristalito, à medida que a concentração de $\mathrm{NaBH}_{4}$ aumenta, Tabelas 1 e $2 . \mathrm{Na}$ Tabela 1 essa diminuição é mais acentuada nos eletrocatalisadores PtRu/C-1(GG), PtRu/C-2(GG) e PtRu/C-5(GG) do que em PtRu/C50(GG), PtRu/C-250(GG). Já na Tabela 2, os eletrocatalisadores $\mathrm{PtRu} / \mathrm{C}-1(\mathrm{AR}), \mathrm{PtRu} / \mathrm{C}-2(\mathrm{AR})$ mostraram pouca diferença no tamanho médio das suas partículas, enquanto que PtRu/C-5(AR) mostrou maior diferença em relação a PtRu/C-1(AR) e PtRu/C-2(AR). Por fim, os eletrocatalisadores PtRu/C-50(AR) e PtRu/C-250(AR) apresentaram o mesmo tamanho médio de seus cristalitos.

Observa-se em ambos os métodos que o tamanho médio dos cristalitos diminui significativamente até alcançar um valor de saturação para a razão 1:50, na qual foram obtidos os menores valores de tamanho de cristalitos, $2,7 \mathrm{~nm}$ no método de gotejamento e 1,8 nm no método de adição rápida.

As Tabelas 1 e 2 mostram também uma diminuição nos parâmetros de rede dos eletrocatalisadores para valores em torno de 3,88 $\AA$, menores que o do cristal de platina que é $3,9156 \AA .{ }^{34}$ A diferença nos valores do parâmetro de rede, em relação à $\mathrm{Pt}$, foi ligeiramente maior para as nanopartículas PtRu obtidas pelo método do gotejamento, o que indica um maior grau de ligamento de Pt-Ru para estes eletrocatalisadores.

O fato dos eletrocatalisadores, obtidos pelo método da adição rápida, terem tamanhos de cristalitos menores do que os obtidos por gotejamento sugere que o despejamento imediato do sal redutor não favorece um maior crescimento dos cristais a partir de um germe de cristalização inicial.

\section{Medidas voltamétricas de eletro-oxidação do etanol}

Nas Figuras 3 e 4 estão sobrepostas as curvas obtidas para as varreduras anódicas referentes à oxidação do etanol sobre os

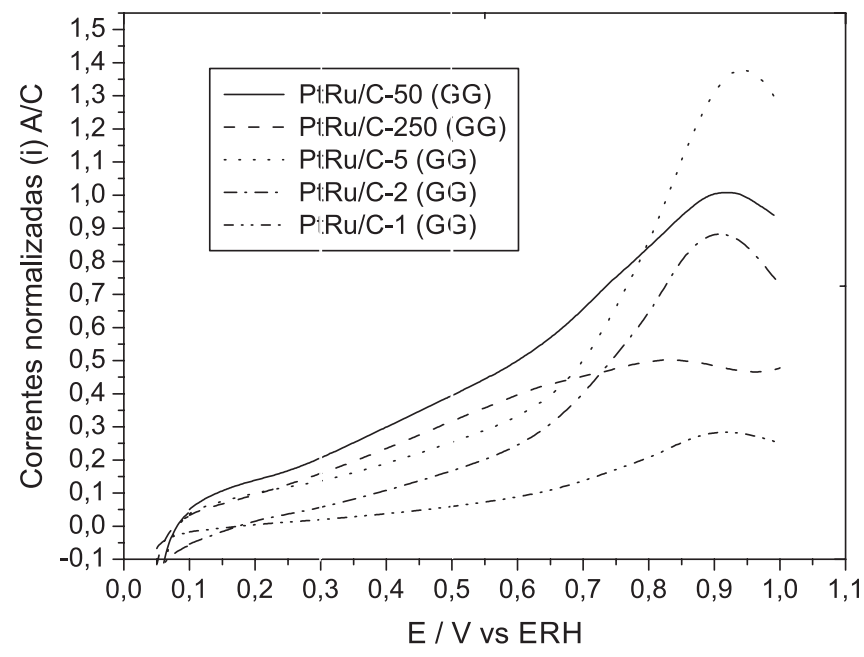

Figura 3. Varreduras anódicas dos eletrocatalisadores PtRu/C-1, PtRu/C-2, PtRu/C-5, PtRu/C-50 e PtRu/C-250 (sintetizados por gotejamento de solução de $\mathrm{NaBH}_{4}$ ) em solução 0,5 mol L de $\mathrm{CH}_{3} \mathrm{CH}_{2} \mathrm{OH}+0,5 \mathrm{~mol} \mathrm{~L}^{-1}$ de $\mathrm{H}_{2} \mathrm{SO}_{4}$, a $20 \mathrm{mV} / \mathrm{s}$

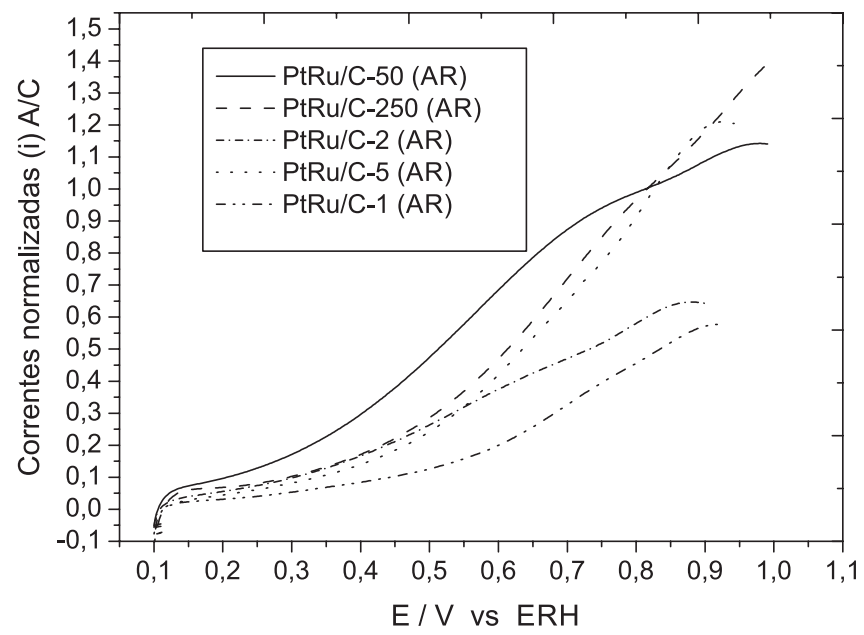

Figura 4. Varreduras anódicas dos eletrocatalisadores PtRu/C-1, PtRu/C-2, PtRu/C-5, PtRu/C-50 e PtRu/C-250 (sintetizados por adição rápida de solução de $\mathrm{NaBH}_{4}$ ) em solução 0,5 mol L-1 de $\mathrm{CH}_{3} \mathrm{CH}_{2} \mathrm{OH}+0,5 \mathrm{~mol} \mathrm{~L} L^{-1}$ de $\mathrm{H}_{2} \mathrm{SO}_{4}$, a $20 \mathrm{mV} / \mathrm{s}$ 
eletrocatalisadores sintetizados por meio do procedimento de adição gota a gota e adição rápida.

As varreduras anódicas presentes nas Figuras 3 e 4 mostraram que para a região de interesse tecnológico $(0,3$ a $0,5 \mathrm{~V})$, os eletrocatalisadores PtRu/C-50(GG), PtRu/C-250(GG), PtRu/C-50(AR) e PtRu/C250(AR) apresentaram valores maiores de corrente normalizada do que os demais. Entretanto, o catalisador PtRu/C-50(AR) possui maior corrente que $\mathrm{PtRu} / \mathrm{C}-50(\mathrm{GG})$ na região considerada. O mesmo não ocorre com PtRu/C-250(AR) que apresentou pior desempenho em relação à PtRu/C-250(GG). Para os outros catalisadores sintetizados por adição rápida, as correntes medidas foram maiores quando comparadas com os catalisadores obtidos com mesma concentração molar no procedimento gota a gota, atestando o efeito benéfico do método de adição rápida e do aumento da concentração da solução de $\mathrm{NaBH}_{4}$, pelo menos até a razão molar 1:50.

Considerando que as respostas dos catalisadores estão normalizadas, não se pode atribuir diretamente a melhora na resposta catalítica ao efeito de aumento de área superficial pela diminuição do tamanho dos cristalitos. Neste caso, deve-se inferir que os cristais menores, obtidos em razões molares (redutor/sais) mais elevadas, apresentam superfícies com sítios catalíticos mais ativos para oxidação do etanol do que as superfícies dos demais catalisadores obtidos com razões molares (redutor/sais) menores.

\section{Medidas cronoamperométricas de eletro-oxidação do etanol}

O desempenho dos eletrocatalisadores para oxidação do etanol foi estudado, também, por cronoamperometria em solução $0,5 \mathrm{~mol}$ $\mathrm{L}^{-1}$ de etanol e $0,5 \mathrm{~mol} \mathrm{~L}^{-1}$ de $\mathrm{H}_{2} \mathrm{SO}_{4}$. Os resultados são mostrados nas Figuras 5 e 6.

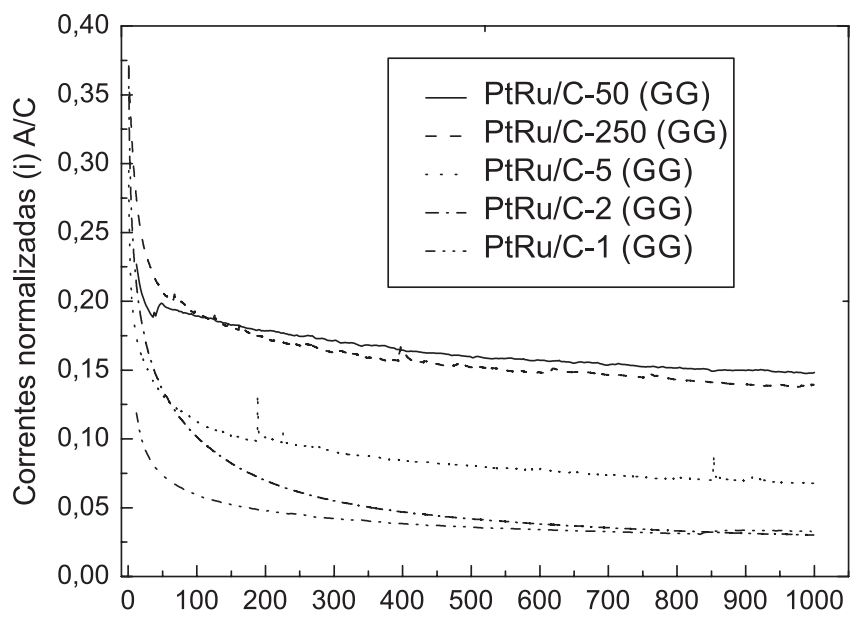

$\mathrm{t}$ (s)

Figura 5. Curvas cronoamperométricas de eletro-oxidação de etanol sobre os eletrodos de PtRu/C-1, PtRu/C-2, PtRu/C-5, PtRu/C-50 e PtRu/C-250 (sintetizados por gotejamento de solução de $\mathrm{NaBH}_{4}$ ) em uma solução de 0,5 mol L ${ }^{-1} \mathrm{CH}_{3} \mathrm{CH}_{2} \mathrm{OH}+0,5 \mathrm{~mol} \mathrm{~L}^{-1}$ de $\mathrm{H}_{2} \mathrm{SO}_{4}$. Potencial aplicado 0,5 $\mathrm{V}$

Observa-se nas curvas cronoamperométricas que ocorre uma rápida diminuição da corrente nos primeiros segundos da reação. Este fato está associado ao mecanismo da oxidação do etanol, que envolve etapas de adsorção química dissociativa e remoção oxidativa dos adsorbatos. ${ }^{26}$ Após os momentos iniciais, existe a tendência de estabelecer um estado quase estacionário, obtendo-se uma corrente que diminui lentamente com o tempo.

Os resultados obtidos com a técnica de cronoamperometria também mostraram que PtRu/C-250(GG) e PtRu/C-50(GG) apresentam valores maiores de corrente normalizada do que os catalisadores

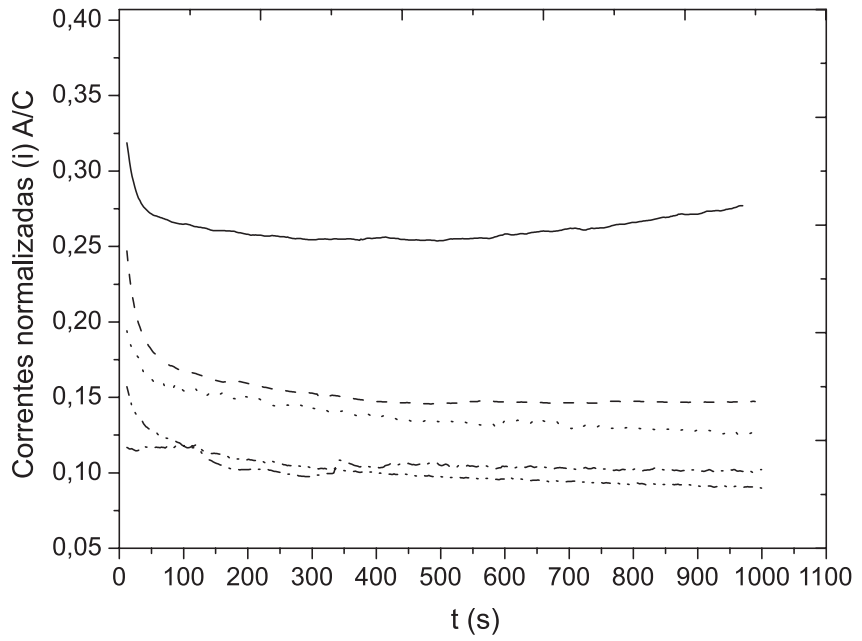

Figura 6. Curvas cronoamperométricas de eletro-oxidação de etanol sobre os eletrodos de PtRu/C-1, PtRu/C-2, PtRu/C-5, PtRu/C-50 e PtRu/C-250 (sintetizados por adição rápida de solução de $\mathrm{NaBH}_{4}$ ) em uma solução de $0,5 \mathrm{~mol} \mathrm{~L}^{-1} \mathrm{CH}_{3} \mathrm{CH}_{2} \mathrm{OH}$ e $0,5 \mathrm{~mol} \mathrm{~L}^{-1}$ de $\mathrm{H}_{2} \mathrm{SO}_{4}$. Potencial aplicado $0,5 \mathrm{~V}$

PtRu/C-1(GG), PtRu/C-2(GG) e PtRu/C-5(GG), após 20 min de reação no potencial de $0,5 \mathrm{~V}$, novamente indicando o efeito benéfico do aumento da concentração de $\mathrm{NaBH}_{4}$ na solução utilizada para redução dos metais. Os cronoamperogramas dos eletrocatalisadores obtidos por adição rápida mostraram que PtRu/C-50(AR) e PtRu/C250(AR) apresentam valores maiores de corrente normalizada que os catalisadores PtRu/C-1(AR), PtRu/C-2(AR) e PtRu/C-5(AR), confirmando PtRu/C-50(AR) como o de maior atividade catalítica. O catalisador PtRu/C-5(AR) apresentou um tamanho de cristalito menor que PtRu/C-2(AR), porém o seu desempenho foi inferior, conforme é observado nas curvas cronoamperométricas da Figura 6. Este resultado não pode ser explicado apenas com as técnicas empregadas neste trabalho. Possivelmente, aspectos relativos à composição atômica superficial estejam associados à causa deste resultado.

Pode-se atribuir, aproximadamente, a seguinte ordem de atividades catalíticas após 20 min de reação: PtRu/C-1(AR) < PtRu/C$5(\mathrm{AR})<\mathrm{PtRu} / \mathrm{C}-2(\mathrm{AR})<\mathrm{PtRu} / \mathrm{C}-250(\mathrm{AR})<\mathrm{PtRu} / \mathrm{C}-50(\mathrm{AR})$. Como se pode observar, PtRu/C-250(AR) não apresentou valores de corrente maiores com o aumento da concentração do sal redutor, sendo o de maior valor PtRu/C-50(AR). Isto indica que a atividade dos catalisadores $\mathrm{PtRu} / \mathrm{C}$ possui uma relação com a concentração do sal redutor, de tal forma que a atividade catalítica apresenta melhor desempenho em determinada concentração deste, não aumentando mais a partir deste valor. As diferenças observadas, em relação aos resultados obtidos por voltametria, podem ser creditadas ao fato de que no método cronoamperométrico as correntes são medidas em estado mais próximo do estado estacionário.

Considerando a aplicação dos eletrocatalisadores em DEFC, a comparação das atividades catalíticas pelo método cronoamperométrico é mais adequada. De qualquer forma, ambos os métodos apontaram que os eletrocatalisadores PtRu/C-50(AR) e PtRu/C-250(AR) apresentaram o melhor desempenho dentre todos os eletrocatalisadores testados.

\section{CONCLUSÕES}

A partir dos resultados de DRX, verificou-se que o tamanho médio dos cristalitos dos eletrocatalisadores nanoparticulados, suportados em carbono e preparados pela metodologia de adição gota a gota da solução de boro-hidreto de sódio, estão na faixa de 2,6 a 4,3 nm. Os eletrocatalisadores preparados por adição rápida apresentaram 
tamanhos médios de seus cristais, na faixa de 1,8 a $3,9 \mathrm{~nm}$, comprovando que o modo de adicionar a solução redutora influencia no tamanho dos cristalitos obtidos.

Os eletrocatalisadores PtRu/C-1(GG), PtRu/C-2(GG) preparados com baixas concentrações de $\mathrm{NaBH}_{4}$ apresentam cristais maiores em comparação com os PtRu/C-5(GG), PtRu/C-50(GG) e PtRu/C250(GG). Este resultado é similar aos obtidos para os eletrocatalisadores obtidos por adição rápida. A análise dos difratogramas também permitiu concluir que ocorreu, em certa extensão, a formação de liga nos eletrocatalisadores sintetizados por ambos os métodos.

A atividade catalítica dos eletrocatalisadores aumenta com o aumento da concentração da solução de $\mathrm{NaBH}_{4}$, até a razão molar de 1:50 metais/boro-hidreto.

Os resultados sugerem que o aumento da atividade catalítica está associado ao menor tamanho dos cristalitos obtidos, tanto pelo método de se adicionar a solução de $\mathrm{NaBH}_{4}$, quanto pelo aumento da razão molar entre $\mathrm{NaBH}_{4}$ e sais precursores. É evidente que a diminuição do tamanho das nanopartículas, por si só, deve ocasionar um aumento na atividade catalítica, por um efeito de aumento da área superficial. No entanto, como as respostas catalíticas foram avaliadas com correntes normalizadas, os resultados indicam que, para a reação de oxidação de etanol em meio ácido, a atividade catalítica intrínseca das nanopartículas de PtRu também aumenta com a diminuição do seu tamanho, pelo menos na faixa de tamanhos médios obtidos neste trabalho.

\section{AGRADECIMENTOS}

Às agências de fomento FINEP e FAPESPA e à Companhia Vale pelos apoios financeiros concedidos.

\section{REFERÊNCIAS}

1. Williams, M. C.; Fuel Cells 2001, 1, 187.

2. Carrette, L. ; Friedirch, K. A.; Stimming, U.; Chem. Phys. 2000, 1, 162.

3. Wan, C.; Chen, C.; Int. J. Hydrogen Energy 2009, 34, 9515.

4. Antolini, E.; J. Appl. Electrochem. 2004, 34, 563.

5. Lopes, P. P; Ticianelli, E. A.; Quim. Nova 2007, 30, 1256.

6. Schimmel, H. G.; Towards a Hydrogen-driven Society?, Delft University Press: Delft, 2004.

7. Zhou, W. J.; Zhou, B.; Li, W. Z.; Zhou, Z. H.; Song, S. Q.; Sun, G. Q.; Xin, Q.; Douvartzides, S.; Goula, M.; Tsiakaras, P.; Appl. Catal. 2003, $126,16$.

8. Oliveira-Neto, A.; Linardi, M.; Spinacé, E. V.; J. Appl. Electrochem. 2006, 17, 309.

9. Song, S.; Tsiakaras, P.; J. Power Sources 2006, 53, 187.
10. Lamy, C.; Belgsir, E. M.; Léger, J. M.; J. Appl. Electrochem. 2001, 31, 799.

11. Brandalise, M.; Dissertação de Mestrado, Instituto de Pesquisas Energéticas e Nucleares, Brasil, 2010.

12. Gonzalez, E. R.; Quim. Nova 2000, 23, 262.

13. Groleau, C.; Coutanceau, C.; Pierre, F.; Léger, J. M.; Electrochim. Acta 2008, 53, 7157

14. Perez, J.; Villullas, H. M.; Gonzalez, E. R.; Quim. Nova 2007, 37, 555.

15. Spinacé, E. V.; Oliveira Neto, A.; Franco, E. G.; Linardi, M.; Gonzalez, E. R.; Quim. Nova 2004, 27, 648.

16. Mukerjee, S.; Urian, R. C.; Electrochim. Acta 2002, 47, 3219.

17. Springer, T.; Zawodzinski, T.; Gottesfeld, S.; J. Electrochem. Soc. 1997, 43,15 .

18. Salgado, J. R. C.; Alcaide, F.; Álvarez, G.; Calvillo, L.; Lázaro, M. J.; Pastor, E.; J. Power Sources 2010, 195, 4022.

19. Watanabe, M.; Motoo, S.; J. Electroanal. Chem. Interfacial Electrochem. 1975, 60, 267.

20. Watanabe, M.; Motoo, S.; J. Electroanal. Chem. Interfacial Electrochem. 1975, 60, 275.

21. Freitas, K. S.; Tese de Doutorado, Instituto de Química de São Carlos, Brasil, 2009.

22. Hoster, H.; Iwasita, T.; Baumgartner, H.; Vielstich, W.; Phys. Chem. Chem. Phys. 2001, 3, 337.

23. Rolison, D. R.; Hagans, P. L.; Swinder, K. E.; Long, J. W.; Langmuir 1999, 15, 774 .

24. McBreen, J.; Mukerjee, S.; J. Electrochem. Soc. 1995, 142, 3399.

25. Camara, G. A.; Giz, M. J.; Paganin, V. A.; TicianelliI, E. A.; J. Electroanal. Chem. 2002, 537.

26. Teixeira, M. E.; Dissertação de Mestrado, Instituto de Química de São Carlos, Brasil, 2007.

27. Antolini, E.; J. Power Sources 2007, 170, 1.

28. Souza, J. P. I.; Tese de Doutorado, Instituto de Química de São Carlos, Brasil, 2000.

29. Rojas, S.; Gárcia, G. F.; Jaras, J.; Martinez, H.; Fierro, J.; Bottonet, M.; Appl. Catal. 2005, 285, 24.

30. Liu, Z.; Ling, X.; Su, X.; Lee, J. Y.; Gan, L. M.; J. Power Sources 2005 , 149,1 .

31. Hyun, M.; Kim, S.; Lee, B.; Peck, D.; Shul, Y.; Jung, D.; Catal. Today 2008, 132, 138

32. Souza, J. P. I.; Iwasita, T.; Nart, F. C.; Vielstich, W.; J. Appl. Electrochem. 2000, 30, 43.

33. Kashyout, A. B.; Abu Nassr, A. B. A. A.; Giorgi, L.; Mayalagan, T.; Youssef, B. A. B.; Int. J. Electrochem. Sci. 2011, 6, 379.

34. Antolini, E.; Cardellini, F.; J. Alloys Compd. 2001, 305, 118. 